\title{
La investigación científica en los hospitales privados
}

\author{
Fiacro Jiménez Ponce ${ }^{1}$
}

El conocimiento científico difiere del conocimiento común en la secuencia metodológica para su obtención y en la probabilidad de ser verificado. Sin embargo, en el pasado se han señalado algunas brechas que limitan el trabajo de investigación científica del médico en México. ${ }^{1}$ En este análisis planteado en el 2016 se señaló que la formación metodológica del estudiante de medicina recae fundamentalmente en las instituciones de educación superior, ya sea en la formación durante la licenciatura o en el postgrado de las residencias médicas. Los hospitales públicos, en ocasiones, desarrollan algún proyecto para incrementar la formación de recursos humanos en ciencia, pero son escasos. Por otro lado, en los hospitales privados la formación de investigadores es aún menos frecuente.

En función de este análisis es necesario plantearse el papel y el desempeño que las instituciones de salud privadas han tenido en el trabajo científico de nuestro país. La formación científica de los médicos, quienes ejercen en los hospitales privados nacionales, generalmente no se promueve. No existen programas específicos encaminados a mejorar las competencias y los conocimientos metodológicos del personal en la mayoría de las instituciones de salud privada. Los mejores esfuerzos consistirán en cursos o diplomados temáticos. Generalmente, los grupos de investigación privados se establecen dentro de los hospitales como centros de investigación para la industria farmacéutica, como áreas de vinculación con instituciones públicas o como unidades no lucrativas de investigación pero siempre como un esfuerzo aislado y generalmente no planeado.

Otro aspecto que debe ser analizado es una discusión sobre la suficiencia y concurso por fondos para investigación que pueden provenir de tres fuentes: las públicas, las privadas y las provenientes de fundaciones. En cuanto

\footnotetext{
1 Jefe de la División de Investigación, Hospital Ángeles Pedregal.
}

Correspondencia:

Fiacro Jiménez Ponce

Correo electrónico: fiacrojimenez@yahoo.com

Aceptado: 08-03-2017.

Este artículo puede ser consultado en versión completa en http:// www.medigraphic.com/actamedica al aspecto de obtención de fondos dedicados a la investigación, como lo señala el Informe General del Estado de la Ciencia, la Tecnología y la Innovación del 2014, editado por el Consejo Nacional de Ciencia y Tecnología, el incremento observado entre el 2008 y el 2014 que en números redondos va de 60 mil a 90 mil millones de pesos aplicados al Gasto Interno en Investigación y Desarrollo Experimental (GIDE) provinieron en dos tercios del sector público y sólo un tercio del sector privado.

La conclusión derivada de esta información parece orientarnos a pensar que la iniciativa privada no ha incrementado su inversión en ciencia desde el 2007. En cambio, la del gobierno federal ha incrementado hasta alcanzar un GIDE que corresponde al $0.39 \%$ del producto interno bruto de nuestro país. Sumado al $0.11 \%$ de GIDE proveniente de la iniciativa privada y a un 0.04 de otras fuentes, México alcanzaría apenas un $0.54 \%$ de PIB aplicado a ciencia y tecnología. ${ }^{2}$ Comparado con el GIDE de los países del continente, excepto Norteamérica, México está en el tercer lugar, donde Brasil aplica el 1.15\% y Argentina el $0.58 \%$. Comparado con los países de la OCDE, México ocupa el noveno lugar de GIDE; el promedio para estos pacientes es de $2.37 \%$.

Muy probablemente, la dificultad para invertir en ciencia y tecnología en México se deba a otras dos circunstancias. Existe también una brecha en transferencia de tecnología, lo que se traduce como una deficiencia en la innovación y en la comercialización de los productos del conocimiento científico y, finalmente, se señala una separación debida a la ausencia de indicadores claros sobre el impacto que la ciencia tiene sobre el bienestar de la población o el mantenimiento del ambiente. La ciencia no sólo es una manera de crear conocimiento, es fundamentalmente un motor de desarrollo y una fuente de recursos. Los países más desarrollados, incluyendo los de la OCDE, en total presentan más del $50 \%$ de los recursos dedicados a investigación provenientes de fondos privados. La generación de patentes comercializables ayuda a convertir a un país en un modelo económico e innovador. Para ello es necesario establecer una cadena productiva entre la ciencia, la industria y la sociedad.

Una alternativa para un modelo de investigación en un hospital privado podría ser el desarrollo de una unidad de investigación autosustentable que combine 
la atención a los proyectos multicéntricos de la industria farmacéutica, que colabore con la formación de recursos humanos en investigación y que desarrolle un plan para obtener la transferencia tecnológica. La Cámara Nacional de la Industria Farmacéutica ha publicado en su página electrónica que los laboratorios productores de medicamentos y dispositivos médicos entre el 2007 y el 2011 han invertido en México 5,144 millones de pesos. ${ }^{3}$ Estos recursos son utilizados en su mayoría en el desarrollo de centros particulares y sólo en un porcentaje, alrededor del $25 \%$, se aplican en instituciones públicas. De tal forma, un programa de desarrollo científico dentro de los hospitales del Grupo Ángeles no sólo es posible sino necesario. La investigación de alta calidad en hospitales privados es una práctica común en los grandes centros hospitalarios de los Estados Unidos de Norteamérica; en México también debe ser una actividad que habrá de estimularse ya que se cuenta con todos los elementos y estructura para realizarla.

\section{REFERENCIAS}

1. Jiménez-Ponce F, Serrano-Berrones MA, Gutiérrez-Vega R. Four gaps in scientific research in health institutions in Mexico. Rev Med Hosp Gen Méx. 2016; 79 (4): 181-182.

2. Informe General del Estado de la Ciencia, la Tecnología y la Innovación. México, 2014. Consejo Nacional de Ciencia y Tecnología. Diciembre, 2015. Disponible en: http://www.siicyt.gob.mx/index. php/transparencia/informes-conacyt/informe-general-del-estadode-la-ciencia-tecnologia-e-innovacion/informe-general-2014/1572informe-general-2014/file

3. http://www.canifarma.org.mx/investigacionydesarrollo.html 